\title{
Evaluation of an automated method for urinocolture screening
}

\author{
Claudia Ballabio', Natascia Venturi', Maria Roberta Sala', Paolo Mocarelli', Paolo Brambilla',2 \\ I Servizio Universitario di Medicina di Laboratorio Ospedale di Desio, Desio \\ 2 DIMS, Facoltà di medicina e Chirurgia, Università Di Milano, Bicocca
}

Key words: Urinary tract infections, Automatization, Urinoculture

Valutazione di un metodo automatizzato per lo screenig delle urinocolture

\section{SUMMARY}

Introduction: Urinary tract infections are one of the most common diseases found in medical practice and are diagnosed with traditional methods of cultivation on plates. In this study we evaluated an automated instrumentation for screening of the urinocultures that can provide results quickly and guarantee traceability.

The comparison of results obtained with automatic and plate methods is reported.

Methods: 316 urine samples including midstream urine, urine catheter and urine bag have been analyzed by Alfred 60 (Alifax) through light scattering technology that measures the replication of the bacteria. Simultaneously, the samples were sown on agar plates CPS3, Agar Cled, Mc Conkey Agar.

Results: A total of 316 samples were analyzed by the automated method, 190 resulted negative, all confirmed by culture, while 126 were found positive. 82 cases were confirmed positive in culture plate, 65 with significant isolation of bacteria and 17 with polymicrobial flora with a significant charge. 44 cases were negative in culture plate but positive for the automated method.

Conclusions: The absence of false negative results at low charges can represent a starting point to introduce an automated method for urinocolture screening.

\section{INTRODUZIONE}

Le infezioni delle vie urinarie (IVU), parallelamente a quelle del tratto respiratorio, rivestono grande rilevanza clinica per l'elevata frequenza con cui vengono riscontrate nella comune pratica medica e per le complicanze che ne derivano. In ambito nosocomiale risultano essere più frequentemente associate a manovre diagnostiche strumentali ed al cateterismo uretrale $(3,4)$.

Esse sono causate principalmente da microorganismi Gram negativi con una prevalenza maggiore di Escherichia coli (70\%) anche se si verificano sempre più frequentemente casi di infezioni complicate causate da batteri Gram positivi o da germi multiresistenti sui quali i comuni agenti antimicrobici risultano inefficaci portando inevitabilmente a fallimenti terapeutici (3).

L'utilizzo dei tradizionali metodi di coltura su piastra prevedono tempi di crescita delle colonie e di refertazione dei campioni negativi di 24 ore e di 48 ore per quelli positivi. Questo discosta dalle esigenze del clinico di avere risultati in tempi rapidi per impostare una terapia mirata evitando l'utilizzo di antibiotici a largo spettro e migliorando così la gestione del paziente. Anche per quanto riguarda pazienti in età pediatrica risulta di rilevante importanza la tempestività del risultato dell'urinocoltura per iniziare la terapia antibiotica adeguata solo se necessario poiché in questi casi i sintomi di infezione delle vie urinarie non risultano sempre specifici.

In secondo luogo i metodi tradizionali presentano diverse problematiche tra cui possibili errori nella manualità, variabilità nella determinazione della carica batterica dovuta alla capacità e all'esperienza del singolo operatore, la tracciabilità difficoltosa dei campioni e dei risultati.

La possibilità di migliorare la risposta a queste problematiche è stata valutata per un metodo fra quelli attualmente in commercio che svolga, con procedura completamente automatizzata, lo screening dei campioni di urinocoltura afferenti al laboratorio.

\section{MATERIALI E METODI}

Nel mese di Giugno 2009 sono stati analizzati un totale di 316 campioni di urinocoltura comprendenti urina da mitto intermedio, urina da catetere, urina da sacchetto, afferenti al settore di microbiologia di pazienti ambulatoriali e ricoverati. Il protocollo prevede il confronto dei risultati ottenuti utilizzando l'analizzatore Alfred 60 (Alifax, Padova, Italy) ed il metodo di semina su piastra.

Alfred60 esegue l'analisi batteriologica delle urine in modo automatico. Il sistema dispensa $500 \mu \mathrm{l}$ di campione in cuvette di lettura dedicate (vials), contenenti brodo eugonico costantemente agitato e termostatato $\left(37^{\circ} \mathrm{C}\right)$. Durante l'incubazione, ogni $5 \mathrm{~min}$, il raggio laser attraversa la cuvetta permettendo al sistema di rilevare le variazioni di torbidità. Lo scatter prodotto viene, infatti, raccolto da due detector, uno posto a $30^{\circ}$ e l'altro a $90^{\circ}$ rispetto al raggio incidente, elaborato dal SW e visualizzato graficamente in tempo reale.

La prima lettura di ogni campione evita che la torbidità iniziale dovuta a sali, eritrociti, leucociti o cellule di sfaldamento o batteri morti interferisca con il risultato finale.

La crescita batterica rilevata dallo strumento è quindi determinata esclusivamente da batteri vivi e replicanti.

Lo strumento fornisce la possibilità di programmare automaticamente da host il cut-off del singolo campione in base alle esigenze diagnostiche del reparto di provenienza e/o del paziente. Durante la sperimentazione è stata utilizzata una soglia fissa preliminare di $800 \mathrm{CFU} / \mathrm{mL}$ ed un tempo di analisi di 3 ore.

Successivamente i 316 campioni sono stati analizzati impiegando i tradizionali metodi di coltura su piastra comunemente utilizzati in laboratorio i quali prevedono la semina di $1 \mu \mathrm{l}$ di urina su terreni solidi quali Agar CPS3, Agar CLED e Agar Mc Conkey (bioMérieux, Marcy l'etoile, France) ed incubazione delle piastre a $37^{\circ}$ per 24 ore. Le identificazioni biochimiche e gli antibiogrammi sono stati eseguiti mediante il sistema Vitek2 (bioMérieux, Marcy l’etoile, France).

Sono stati quindi confrontati i risultati ottenuti con i due metodi, su piastra ed automatizzato.

\section{RISULTATI}

Su un totale di 316 campioni analizzati con il metodo automatizzato utilizzando una soglia di $800 \mathrm{CFU} / \mathrm{mL}, 190$ sono risultati negativi (60\%), tutti confermati dalla semina su pia-

\section{Corresponding author: Claudia Ballabio}

Servizio Universitario di Medicina di Laboratorio, Ospedale di Desio

Via Mazzini I, 20033 Desio (MI) - Tel. 0362383296

E-mail: cballabio@uds.unimib.it 
stra mentre 126 sono risultati positivi (40\%). 82 campioni sono stati confermati positivi in coltura su piastra, 65 (51\%) con isolamento del microorganismo patogeno e 17 (13\%) aventi flora polimicrobica con carica significativa. 44 casi (35\%) sono risultati invece negativi o con carica inferiore alla soglia di significatività alla coltura su piastra.

Per l'elevata torbidità iniziale rilevata, due campioni sono stati seminati su piastra come consigliato dal produttore. In Tabella 1 sono riportate le soglie con i corrispondenti valori di sensibilità, specificità, valore predittivo positivo e negativo. In Figura I è rappresentata la curva ROC relativa al metodo in esame che presenta un'area di 0.89 .

Le prevalenze relative ai microrganismi isolati dalla coltura sono mostrate in tabella 2.

Analogamente a quanto accade per i batteri, lo strumento ha determinato la positività anche in casi di infezioni da Candida albicans e Candida glabrata.

Analizzando i risultati ottenuti aumentando la soglia di sensibilità si osserva che, portando il cutoff a 9000 UFC/ml, non si riscontra alcun risultato falsamente negativo. Infine variando il cut off da 800 a 9000, il numero dei campioni seminati decresce da 126 a 108.

\section{DISCUSSIONE}

I sistemi tradizionali di coltura su piastra presentano diverse problematiche che potrebbero trovare risoluzione attraverso l'impiego in microbiologia del sistema automatizzato preso in esame nel presente studio.

La possibilità di ottimizzare il valore soglia in base alle esigenze del laboratorio senza perdere in specificità, gli ottimi valori di sensibilità, l'elevato valore predittivo negativo, l'ottimo valore dell'area ottenuta dalla curva ROC, rendono interessante l'introduzione del metodo automatizzato per lo screening delle urinocolture che permette quindi di poter discriminare positività o negatività dei campioni in tre ore refertando in giornata i campioni negativi.

Risulta interessante osservare che, modificando adeguatamente la soglia di sensibilità a $9000 \mathrm{CFU} / \mathrm{mL}$ lo strumento è in grado di rilevare tutti i campioni veri positivi escludendo quelli aventi flora polimicrobica da inquinamento (bassa carica) senza evidenza di infezione. Questo potrebbe essere spiegato dal fatto che i microrganismi contaminanti presentano una LAG phase (o fase di adattamento) di circa 3h incominciando a replicarsi, quindi, proprio al termine dell'analisi. Inoltre le prevalenze dei germi isolati sono in linea con il trend normalmente riscontrato nelle urinocolture con una percentuale maggiore di Escherichia coli. Per favorire una maggior comprensione del quadro clinico ed evitare referti falsamente negativi, il sistema può effettuare, contemporaneamente al test colturale, la misura del potere antimicrobico residuo (P.A.R. test) che permette di rilevare nel campione la presenza di possibili inibitori della crescita quali farmaci o sostanze con proprietà antibatteriche(15). Inoltre, la possibilità di integrare al sistema l'esecuzione di un antibiogramma clinico messo a punto secondo le linee guida Cumitech e CLSI (Clinical Standard Laboratory Institute) ed eseguibile in tre ore permetterebbe di ottimizzare ulteriormente il flusso di lavoro con conseguente refertazione in tempi più rapidi anche dei campioni positivi $(6,12)$. Il processo di analisi è ottimizzato e standardizzato, il sistema è pulito e sicuro con una sostanziale riduzione del rischio di esposizione per l'operatore rispetto a quello che si avrebbe con l'utilizzo delle semine su piastra. La tracciabilità del campione è assicurata dalla lettura del codice a barre e la lettura automatizzata delle curve di crescita riduce la possibilità di errore dovuto alla manualità e all'interpretazione sog-

Figura I. Curva ROC per la valutazione del metodo automatico Alfred60. (

Tabella I. Numero di campioni falsi positivi (FP), falsi negativi (FN), veri positivi (VP), veri negativi (VN); valore percentuale di sensibilità (\% SENS), specificità (\% SPEC), valore predittivo negativo (VPN\%), valore predittivo positivo (VPP\%) ottenuti variando il cutoff.

\begin{tabular}{ccccccccc}
\hline \multicolumn{2}{l}{ specificita $(\%$ SPEC), valore predittivo negativo (VPN\%), valore predittivo positivo (VPP\%) } & ottenuti variando il cutoff. & \\
\hline SOGLIA & FP & FN & VP & VN & \%SENS & \%SPEC & VPN\% & VPP\% \\
\hline $\mathbf{8 0 0}$ & 44 & 0 & 82 & 190 & 100 & 81.2 & 100 & 65 \\
\hline $\mathbf{2 0 0 0}$ & 36 & 0 & 82 & 198 & 100 & 84.6 & 100 & 69.5 \\
\hline $\mathbf{6 0 0 0}$ & 26 & 0 & 82 & 208 & 100 & 88.8 & 100 & 75.9 \\
\hline $\mathbf{9 0 0 0}$ & 24 & 0 & 82 & 210 & 100 & 89.7 & 100 & 77.3 \\
\hline $\mathbf{1 5 0 0 0}$ & 9 & 1 & 81 & 225 & 98.7 & 96.1 & 99.5 & 90.1 \\
\hline $\mathbf{3 0 0 0 0}$ & 9 & 1 & 81 & 225 & 98.7 & 96.1 & 99.5 & 90.1 \\
\hline
\end{tabular}

Tabella 2. Numero e percentuale di microrganismi isolati sul totale dei campioni di urinocoltura positivi con germe patogeno isolato

\begin{tabular}{|c|c|c|}
\hline SPECIE ISOLATA & NUMERO ISOLATI & PERCENTUALE ISOLATI \\
\hline Escherichia coli & 47 & 72.4 \\
\hline Proteus mirabilis & 6 & 9.2 \\
\hline Klebsiella pneumoniae & 4 & 6.2 \\
\hline Enterococcus faecalis & 2 & 3 \\
\hline Candida glabrata & 2 & 3 \\
\hline Candida albicans & 2 & 3 \\
\hline Enterobacter aerogenes & $I$ & 1.6 \\
\hline Providencia stuartii & I & 1.6 \\
\hline TOTALE & 65 & 100 \\
\hline
\end{tabular}


gettiva dei risultati.

Inoltre l'automazione dell'intero processo di analisi permette di ottimizzare la gestione del lavoro in laboratorio diminuendo l'operatività del personale dedicato alla batteriologia delle urine ed aumentando il numero di analisi effettuabili in giornata.

L'unica limitazione riscontrata al sistema è rappresentata dalla difficoltà di lettura dei campioni con un elevato grado di torbidità; per questa ragione è consigliabile procedere alla semina su piastra dei campioni indicati dal sistema come “torbido" poiché in essi è altamente probabile la presenza di flora batterica.

\section{BIBLIOGRAFIA}

1. Breda E. Principles and thecnology of the Uro-Quick system for bacteriuria rapid screening. Galeno 1996; 4: 11-21.

2. Cavallo MR, Carpi D, Peyronel E, et al. Alfred60 -Hb\&L automatic instrument for the urine culture evaluation: managerial aspects. Poster $22^{\circ}$ National Conference SIMeL, Rimini 28-31October 2008.

3. Fontana C, Favaro M, Minelli S, et al. A novel culturing system for fluid samples. Med Sci Monit, 2009; 15: BR55-60.

4. Foxman B. Epidemiology of urinary tract infections incidence, morbidity, and economic costs. Dis Mon, 2003; 49: 53-70.

5. Gould C, Allen-Bridson K, Horan T. Surveillance definitions for urinary tract infections. Clin Infect Dis, 2009; 49: 1288-9.

6. Huang TD, Debecker C, Simon A. Evaluation of the automated urine culture system Alfred60 for the screening of significant bacteriuria. poster $18^{\text {Th }}$ ECCMID, Barcelona 19-22 April 2008.

7. ISO 17025: 2005, General requirements for the competence of testing and calibration laboratories.

8. ISO 9000: 2000 Sistemi di gestione per la qualità- Fondamenti e terminologia.

9. Laneve M, Mazzone C. Urinary tract infections: etiology and antimicrobial susceptibility of uropathogens. Microb Med, 2009; 24 (1): 36-9.

10. Pezzati E, Marengo S, Roveta S, et al. Evaluation of the Uro-Quick system for antibiotic susceptibility tests of strains collected from intensive care units. Annal of Microbiology, 2006; 56: 179-83.

11. Raggi M, Soro O, Schito GC. Performance of a new automated method for the detection of bacteriuria. Abstract, 7th European Congress of Clinical Microbiology and Infectious Diseases, Vienna, March 26-30, 1995.

12. Ricci L. L'automazione delle urinocolture nuovi percorsi diagnostici ed organizzativi. SIMPIOS, Grado, 7-9 April 2008.

13. Roveta S, Debbia EA, Marchese A. The Uro-Quick System For the identification of Well Characterized Antibiotic resistance Strains. Microb Med, 2003; 18: 49-55.

14. Roveta S, Marchese A, Debbia EA. Antibiotic Susceptibility tests directly on urine samples by Uro-Quick, a rapid automated system. J Chemother, 2006: 12-9.

15. Squarzon L, Cavallaro. Utility of a rapid method for the culture of human biological liquids from sterile districts. Poster AMCLI, Stresa, 5-8 ottobre 2008.

16. Trautner BW. Management of catheter-associated urinary tract infections. Curr Opin Infect Dis, 2010: 76-82. 